\title{
Emergency department use is high after esophagectomy and feeding tube problems are the biggest culprit
}

Biniam Kidane, MD, MSc, ${ }^{\text {a,b }}$ Suha Kaaki, MD, ${ }^{a}$ Dhruvin H. Hirpara, MD, ${ }^{\mathrm{b}}$ Yu Cindy Shen, BSc, Adam Bassili, MD, ${ }^{b}$ Frances Allison, BSc, ${ }^{c}$ Thomas K. Waddell, MD, PhD, ${ }^{b, c}$ and Gail E. Darling, MD $^{\mathrm{b}, \mathrm{c}}$

\section{ABSTRACT}

Objectives: Esophagectomy is a complex operation with potential for prolonged recovery. We aimed to identify the incidence of and risk factors for any and frequent emergency department visits within 1 year of esophagectomy.

Methods: A retrospective cohort study was performed looking at consecutive esophagectomies at a tertiary Canadian center (1999-2014). Multivariable analyses identified factors associated with any emergency department visits and frequent emergency department use ( $\geq 3$ visits) within 1 year postesophagectomy.

Results: There were 520 esophagectomies with in-hospital mortality of $6 \%$ $(n=31)$. Of those discharged, 29.7\% $(n=145)$ had $\geq 1$ emergency department visit. Most common causes were feeding tube problems $(39.3 \% ; n=57)$ and dysphagia/stricture $(13.1 \% ; \mathrm{n}=19)$. Higher income (adjusted odds ratio [aOR], 1.22; 95\% confidence interval [CI], 1.04-1.42 per $\$ 10,000)$ and use of hybrid/minimally invasive esophagectomy (aOR, 3.24; 95\% CI, 1.71-6.11) were independently associated with having emergency department visits. Patients with hybrid/minimally invasive esophagectomy were discharged earlier than others $(P<.0001)$. Living outside of our metropolitan area $(\mathrm{aOR}, 0.36 ; 95 \%$ CI, 0.27-0.49) and having surgery in the later years of the study period (aOR, $0.91 ; 95 \%$ CI, 0.86-0.97; $P=.006$ ) were both independently associated with lower odds of emergency department visits. Forty-three patients $(8.8 \%)$ were frequent emergency department users, with the most common causes of repeat emergency visits being feeding tube problems. Living outside of our metropolitan area was associated with lower odds of frequent emergency visits (aOR, 0.25; $95 \%$ CI, 0.14-0.45).

Conclusions: There is high emergency department use within 1 year postesophagectomy. Patients living farther away from our hospital had a lower rate of emergency department use. It is possible that they are utilizing emergency departments nearer to home; this needs further study. Feeding tube problems are the biggest culprits and are potentially modifiable. (J Thorac Cardiovasc Surg 2018;156:2340-8)

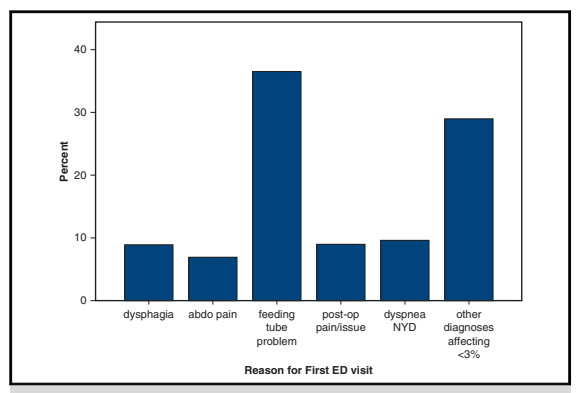

Reasons for first emergency department visit.

\section{Central Message}

Emergency room use is high at 1 year after esophagectomy with rates of $30 \%$ use and $9 \%$ frequent visits. Feeding tube issues were the common causes of emergency room use.

\section{Perspective}

There is a gap in the literature regarding emergency department use after esophagectomy, despite its influence on costs and patient quality of life. Emergency use is high at 1 year after esophagectomy and feeding tube issues were the common causes of emergency room use. Quality improvement strategies that address the identified risk factors can potentially reduce emergency room use and improve quality. Anticipatory information and services for patients with feeding tubes may be helpful.

See Editorial Commentary page 2349

See Editorial page 2338 .
From the a Section of Thoracic Surgery, University of Manitoba, Winnipeg, Manitoba, Canada; 'bivision of Thoracic Surgery, University of Toronto, Toronto, Ontario, Canada; and ${ }^{\mathrm{c} D}$ Division of Thoracic Surgery, Toronto General Hospital, Toronto, Ontario, Canada.

Funding support was provided by the Kress Family Chair in Esophageal Cancer.

Read at the 43rd Annual Meeting of The Western Thoracic Surgical Association, Colorado Springs, Colorado, June 21-24, 2017.

Received for publication July 13, 2017; revisions received June 16, 2018; accepted for publication July 9, 2018; available ahead of print Oct 8, 2018

Address for reprints: Biniam Kidane, MD, MSc, Health Sciences Centre, Room GE611, Winnipeg, Manitoba, Canada R3A (E-mail: b.kidane@mail.utoronto.ca). $0022-5223 / \$ 36.00$

Copyright (c) 2018 Published by Elsevier Inc. on behalf of The American Association for Thoracic Surgery

https://doi.org/10.1016/j.jtcvs.2018.07.100
Surgery is the primary treatment for operable cancers of the esophagus either alone or combined with chemotherapy or chemoradiation therapy. ${ }^{1,2}$ Esophagectomy is amongst the most morbid of surgical procedures and can result in complications that may persist for sustained periods after

Scanning this QR code will take you to the article title page. 


\section{Abbreviations and Acronyms \\ $\mathrm{ED}=$ emergency department \\ $\mathrm{CCI}=$ Charlson comorbidity index \\ MIS $=$ minimally invasive \\ LOS $=$ length of stay}

surgery or arise in a delayed fashion. ${ }^{3-5}$ This has implications on quality of life of patients as well resource use. ${ }^{6}$ Emergency department (ED) visits following the operation are common and have consequences in terms of health resource use and quality of life.$^{6-9}$ Although there exist data about readmissions after esophagectomy, there is a significant gap in the literature on the incidence and major causes of ED visits after esophagectomy. This gap in the literature is important to address because isolated and frequent ED use can have significant influence on health care costs as well as the quality of life of vulnerable patients whose quality of life has already been compromised by their esophageal cancer and its treatment. As part of a quality improvement initiative, we aimed to identify the incidence of ED visits as well as investigate the reasons for any and frequent ED visits. This would hopefully allow us to design targeted interventions to reduce the burden of ED visits both for our patients and our health care system.

To date, all studies of ED visits have evaluated a time window of 30 days after discharge from esophagectomy hospitalization. Because esophagectomy has longer-term effects, limiting evaluation of ED visits to 30 days likely underestimates the true burden of the problem. The primary objectives of this study were to determine the incidence of any and frequent ED visits within 1 year of esophagectomy as well as to identify factors associated with increased ED use.

\section{METHODS}

This study was approved by the University Health Network Research Ethics Board. Requirement for patient consent was waived due to the retrospective nature of the analysis. Using data from a prospectively collected esophageal cancer database, we conducted a retrospective cohort study of consecutive esophagectomies for cancer in a high-volume tertiary hospital in Ontario, Canada, from 1999 to 2014. Reasons for ED visits were assessed by chart review. The outcomes of interest were any ED visits within 1 year of esophagectomy and frequent ED use (defined as $\geq 3$ visits) within 1 year of esophagectomy. For univariable analysis, normally distributed continuous data were reported as means with standard deviations and analyzed using independent sample $t$ tests. Data that were not normally distributed were reported as medians with interquartile ranges and analyzed using the Mann-Whitney $U$ test. Fisher exact tests were used for univariate analysis of categorical data. Hierarchical multivariable logistic regression was used to identify factors independently associated with higher odds of ED visits. Hierarchy was defined by using a forced-entry method as the first step for the following factors that were identified a priori as being important: age, sex, income, comorbidity, resection type, use of hybrid/minimally invasive surgery [MIS], use of chemotherapy and/or radiation therapy, and year of surgery. In the second step of the hierarchy, we included the following factors: presence of social supports, ability to speak English, presence of specific complication types (eg, respiratory, cardiac, thromboembolic, anastomotic, chylothorax, and recurrent laryngeal nerve palsy) that occurred during the index operation hospital stay. We also included any factor that had a $P$ value $<.2$ on univariable analysis. We applied backward stepwise elimination to this second step of the hierarchical logistic regression analysis. Income was estimated based on the median family income of the individual's post code using Canadian Census tract data that has been shown to be a robust and reliable estimation of income. ${ }^{10}$ Comorbidity status was defined using the Charlson comorbidity index $(\mathrm{CCI})$ and was stratified as low $(\mathrm{CCI}=0-2)$ and high $(\mathrm{CCI} \geq 3)$. This stratification was based on the distribution of patients and also fit within usual patterns of categorization in the literature. Year of surgery was dichotomized into 1999 to 2006 and 2007 to 2014 because it created 2 approximately equal periods of time and also because provincial regionalization efforts were enacted in the transition period between those 2 eras. Patients were classified as having social support if they lived with a partner or an adult (aged $>18$ years) child. Multivariable logistic regression was also used to identify factors independently associated with higher odds of frequent ED visits. Due to lower event rates, we used forced entry of any factor that had a $P$ value $<.2$ on univariable analysis. Competing risk analysis was not used for the competing risks of ED visits and mortality after hospital discharge. Although it is true that experiencing death at home after discharge prevents a patient from presenting to the ED, our purpose was to identify the burden of resource use incurred by ED visits; thus, competing risk analysis is not necessary because patients who experience home death after discharge would not be expected to increase the burden of ED visits or resource use. A 2-sided alpha of 0.05 was used for all tests of significance. Statistical analyses were performed using SPSS version 23 (IBM-SPSS Inc, Armonk, NY). Our standard feeding tube technique involved a $16 \mathrm{~F}$ red rubber catheter that was inserted in an open technique. Witzel technique was used for a distance of $2 \mathrm{~cm}$. The jejunum was sutured to the anterior abdominal wall with interrupted sutures circumferentially around the tube and longitudinally to fixate the jejunal limb to prevent twisting. For minimally invasive esophagectomy, we made a small, $3-\mathrm{cm}$ incision in the abdomen to accomplish this. Tubes were flushed twice daily and after feeds or medications. Feeding tubes were removed 6 weeks postoperatively if not being used for nutrition.

\section{RESULTS}

There were 520 esophagectomies performed during the study period with in-hospital mortality of $6.0 \%(n=31)$. Distribution of esophagectomy type was transhiatal $(\mathrm{n}=83)$, McKeown $(\mathrm{n}=108)$, Ivor Lewis $(\mathrm{n}=189)$, left thoracoabdominal $(\mathrm{n}=128)$, or pharyngolaryngectomy $(\mathrm{n}=12)$. Approximately $20 \%$ of these $(\mathrm{n}=107)$ had at least a thoracoscopic or laparoscopic component and were classified as hybrid/minimally invasive. Use of MIS approaches increased significantly in the latter half of the study period, rising from $4.4 \%$ of cases to $40 \%$ of cases $(P<.001)$. Median length of stay was 14 days (interquartile range [IQR], 11-23). Of those surviving to discharge, $29.7 \%$ of patients $(n=145)$ had $\geq 1$ ED visit within 1 year of discharge. Of all ED visits, $30.4 \%$ resulted in eventual readmission to hospital during that visit; thus, the majority were discharged home from the ED. Figure 1 shows the reasons for the first ED visit with the most common cause being feeding tube problems. Many of the feeding tube problems appear to be related to tube blockages or unsubstantiated concerns 


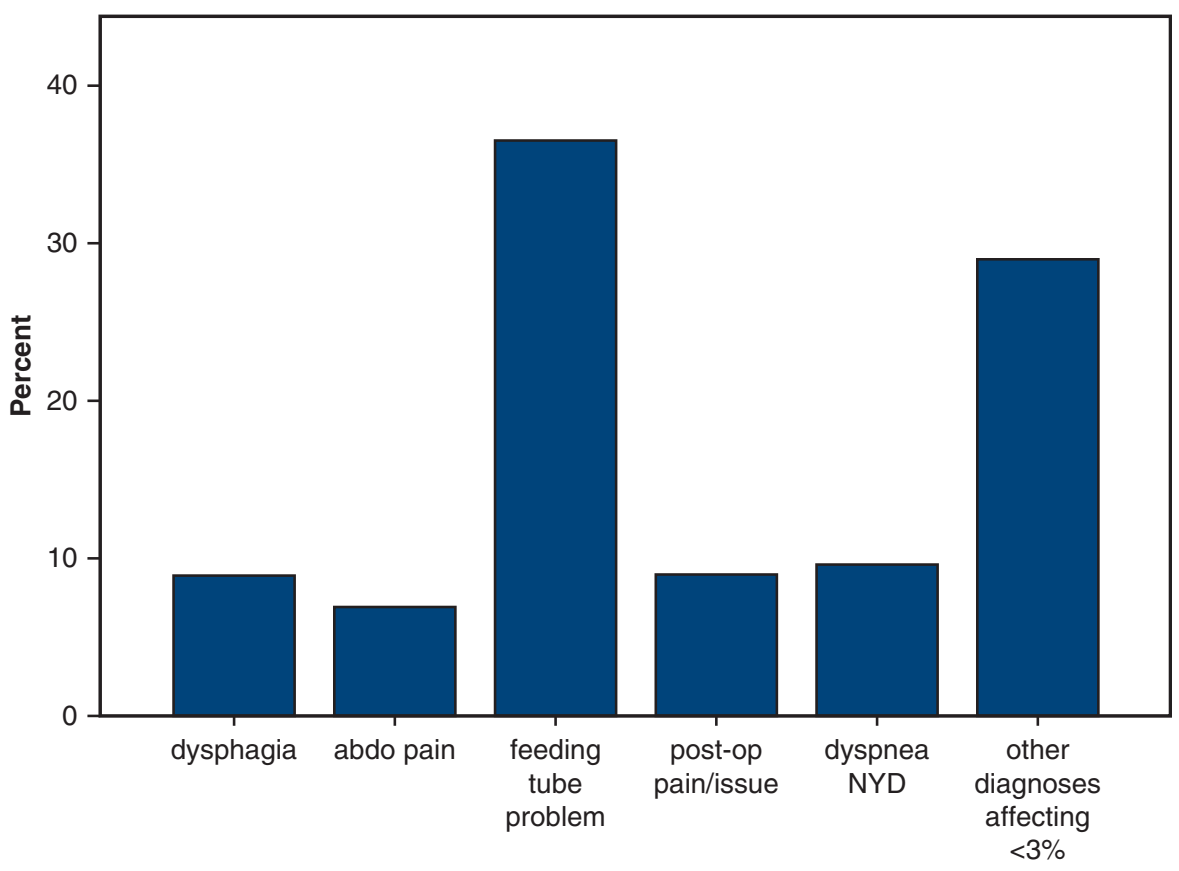

Reason for First ED visit

FIGURE 1. Reasons for first emergency department $(E D)$ visit $(\mathrm{n}=145)$. $N Y D$, Not yet diagnosed.

about infection. Figures 2 and 3 show the reasons for the second, third, and fourth ED visits wherein feeding tube problems remain the most common cause for ED visits. Figure 4 depicts time to first ED visit (in days); the median time to the first ED visit is 18 days after discharge (IQR, 9-56 days).

Table 1 depicts the univariable analysis of differences in baseline characteristics between patients with and without ED visits within 1 year of esophagectomy. On multivariable analysis (Table 2), higher income (adjusted odds ratio
[aOR], 1.22; 95\% confidence interval [CI] 1.04-1.42; $P=.01)$ and use of hybrid/minimally invasive esophagectomy (aOR, 3.24; 95\% CI, 1.71-6.11; $P<.0001$ ) were independently associated with higher odds of ED visits. Patients having a hybrid/minimally invasive component in the esophagectomy were usually discharged earlier than others (median length of stay [LOS], 12 days; IQR, 9-20 days vs median, 15 days; IQR, 11-23 days; $P<.0001)$. Living outside of the greater metropolitan area of our hospital (aOR, 0.39; 95\% CI, 0.29-0.52; $P<.0001)$ and having

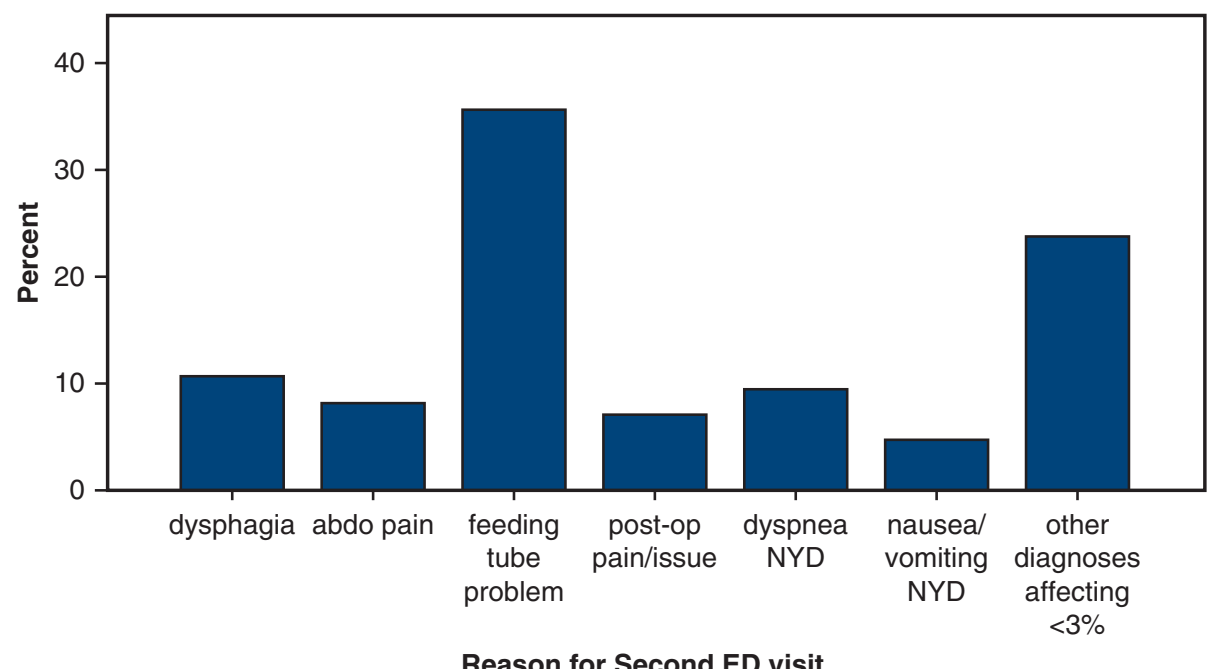

FIGURE 2. Reasons for second emergency department $(E D)$ visit $(\mathrm{n}=84)$. $N Y D$, Not yet diagnosed. 

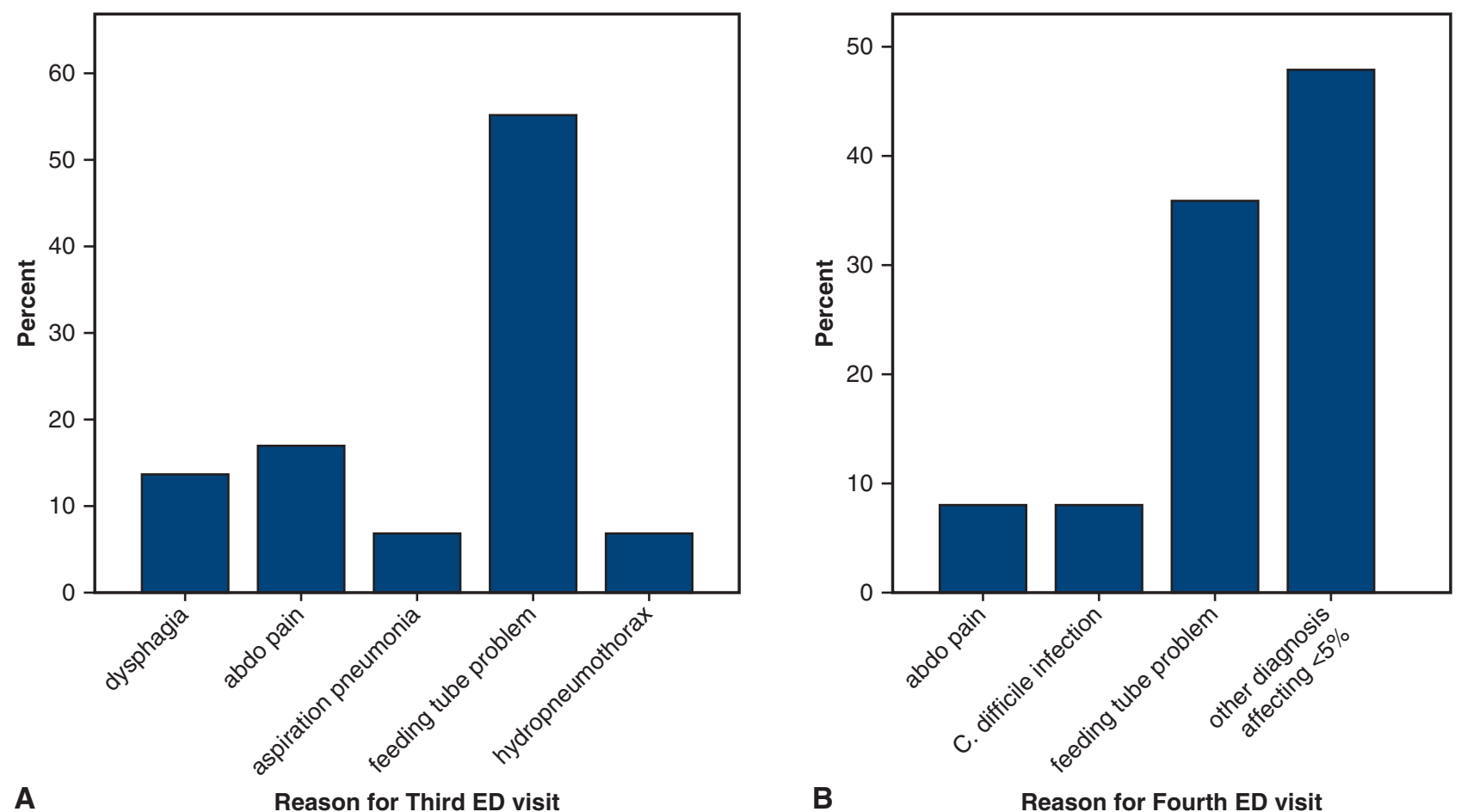

FIGURE 3. A, Reasons for third emergency department $(E D)$ visit $(\mathrm{n}=29)$. B, Reasons for fourth ED visit $(\mathrm{n}=25)$.

surgery in the later years of the study period (aOR, 0.91; 95\% CI, 0.86-0.97; $P=.006$ ) were both independently associated with lower odds of ED visits (aOR, 0.39; $95 \%$ CI, 0.29-0.52; $P<.0001)$.
Forty-three patients $(8.8 \%)$ were frequent ED users, with the most common causes of repeat ED visits being feeding tube problems. Table 3 depicts the univariable analysis of differences between frequent ED users and those who

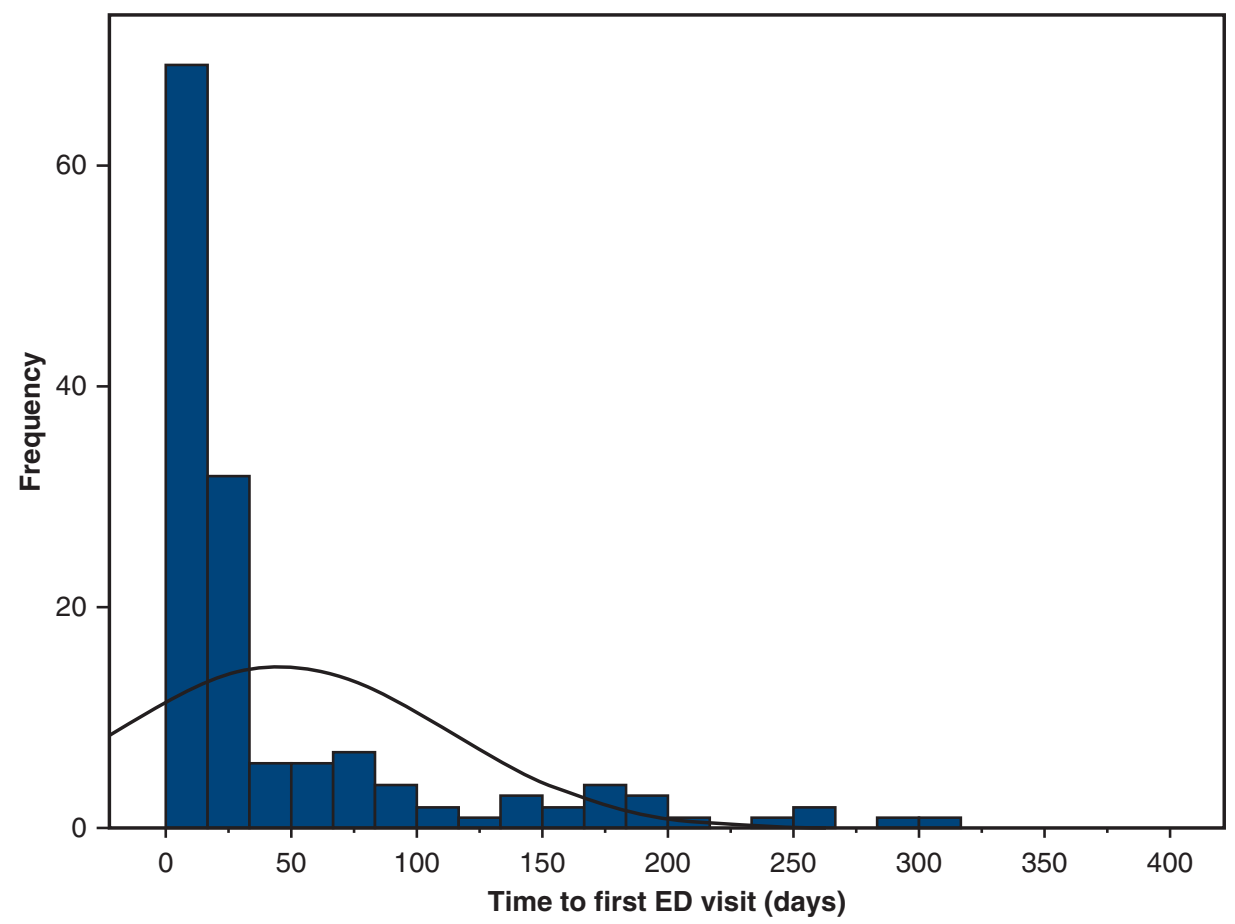

FIGURE 4. Time to first emergency department $(E D)$ visit. 
TABLE 1. Baseline characteristics (emergency department [ED] visits vs no ED visits)

\begin{tabular}{|c|c|c|c|}
\hline Risk factor & $\begin{array}{c}\text { ED visit } \\
(n=145)\end{array}$ & $\begin{array}{l}\text { No ED visits } \\
\quad(n=344)\end{array}$ & $P$ value \\
\hline Year of surgery & & & .24 \\
\hline 1999-2006 & $55.2(80)$ & $49.1(169)$ & \\
\hline $2007-2014$ & $44.8(65)$ & $50.9(175)$ & \\
\hline Age at time of surgery & $61.9 \pm 11.9$ & $62.7 \pm 11.7$ & .45 \\
\hline Male sex & $75.9(110)$ & $75.6(260)$ & 1.00 \\
\hline Median household income (\$) & $77,137(63,464-93,115)$ & $69,546(59,734-87,406)$ & .01 \\
\hline Presence of social support, \% (n) & $82.1(119)$ & $85.8(295)$ & .34 \\
\hline English speaking, \% (n) & $91.7(133)$ & $92.4 \%(318)$ & .85 \\
\hline Residence distance from index hospital & & & $<.0001$ \\
\hline Within greater metropolitan area & $90.0(129)$ & $63.1(217)$ & \\
\hline Other city within $200 \mathrm{~km}$ of index hospital & $9.6(14)$ & $32.8(113)$ & \\
\hline Other city $\geq 350 \mathrm{~km}$ of index hospital & $1.4(2)$ & $4.1(14)$ & \\
\hline Esophagectomy type & & & .98 \\
\hline Transhiatal & $16.6(24)$ & $15.4(53)$ & \\
\hline McKeown & $20.7(30)$ & $20.3(70)$ & \\
\hline Ivor Lewis & $35.2(51)$ & 37.5 (129) & \\
\hline Left thoracoabdominal & $25.5(37)$ & $25.0(86)$ & \\
\hline Pharyngolaryngectomy & $2.1(3)$ & $1.7(6)$ & \\
\hline Lower Charlson comorbidity index* & $69.0(100)$ & $71.2(245)$ & .75 \\
\hline Chemotherapy & $25.5(37)$ & $29.6(102)$ & .38 \\
\hline Radiation therapy & $23.4(34)$ & $25.9(89)$ & .65 \\
\hline Hybrid/minimally invasive & $29.6(43)$ & $18.6(64)$ & .008 \\
\hline Hospital length of stay (d) & $14(11-26)$ & $14(11-21)$ & .76 \\
\hline Respiratory complications & $25.5(37)$ & $20.3(70)$ & .20 \\
\hline Anastomotic leak & $19.3(28)$ & $14.0(48)$ & .14 \\
\hline Laryngeal nerve complication & $9.0(13)$ & $4.9(22)$ & .10 \\
\hline Thromboembolic complication & $2.8(4)$ & $4.1(11)$ & .60 \\
\hline Cardiac complication & $12.4(18)$ & $11.6(39)$ & .88 \\
\hline Chylothorax & $5.5(8)$ & $5.8(20)$ & 1.00 \\
\hline
\end{tabular}

Values are presented as $\mathrm{n}(\%)$, mean \pm standard deviation, or median (interquartile range). Boldface indicates statistical significance. ${ }^{*}$ Charlson comorbidity index 0 - 2 versus $>3$.

were not frequent users. On multivariable analysis (Table 2), the only predictor that was independently associated with frequent ED visits was living outside of the greater metropolitan area, which was associated with lower odds of frequent ED visits (aOR, 0.25, 95\% CI, 0.14-0.45; $P<.0001)$. Sensitivity analyses using the same multivariable analyses but including only patients living in the regions within $100 \mathrm{~km}$ of our hospital revealed the same finding (Table 2).

\section{DISCUSSION}

We aimed to identify the incidence of and risk factors for ED use after esophagectomy. One-third of patients undergoing esophagectomy have 1 or more ED visits within 1 year of being discharged home. Feeding tube problems and dysphagia were the most common and consistent causes for any and frequent ED visits. Many of the feeding tube problems appear to be related to tube blockages or unsubstantiated concerns about infection.

Having an MIS component in the esophagectomy was independently associated with higher odds of ED visits but not frequent ED visits. Patients with an MIS component to their esophagectomy were usually discharged earlier than others. Having surgery during the later years of the study period was independently associated with lower odds of ED visits. These findings may reflect the effects of the learning curve of MIS esophagectomy over time. Use of MIS approaches increased significantly in the latter half of the study period. The association between MIS esophagectomy and ED visits may have been mediated via multiple potential mechanisms. First, it is possible we were sending patients home too early without sufficient 
TABLE 2. Results of multivariable regression analyses showing factors independently associated with any emergency department (ED) use and frequent ED use within 1 year of esophagectomy

\begin{tabular}{|c|c|c|c|c|}
\hline Risk factor & Adjusted odds ratio & \multicolumn{2}{|c|}{ 95 $\%$ Confidence interval } & $P$ value \\
\hline \multicolumn{5}{|l|}{ Any ED use } \\
\hline Year of surgery (reference, 1999-2006) & 0.91 & 0.86 & 0.97 & .006 \\
\hline Age at time of surgery per year increase & 1.00 & 0.98 & 1.02 & .89 \\
\hline Male sex & 0.76 & 0.46 & 1.26 & .29 \\
\hline Median household income (per $\$ 10,000$ ) & 1.22 & 1.04 & 1.42 & .01 \\
\hline Laryngeal nerve complication & 1.73 & 0.95 & 2.20 & .21 \\
\hline Living outside of greater metropolitan area & 0.36 & 0.27 & 0.49 & $<.001$ \\
\hline Charlson comorbidity index (reference, $0-2$ ) & 1.02 & 0.63 & 1.63 & .95 \\
\hline Hybrid/minimally invasive & 3.24 & 1.71 & 6.11 & $<.001$ \\
\hline Respiratory complications & 1.63 & 0.96 & 2.77 & 0.07 \\
\hline Anastomotic leak & 1.60 & 0.88 & 2.90 & .12 \\
\hline \multicolumn{5}{|l|}{ Frequent ED use } \\
\hline Hospital length of stay & 1.01 & 0.99 & 1.02 & .30 \\
\hline Chemotherapy & 0.48 & 0.06 & 3.87 & .49 \\
\hline Radiation therapy & 1.21 & 0.14 & 10.76 & .86 \\
\hline Living outside of greater metropolitan area & 0.25 & 0.14 & 0.45 & $<.001$ \\
\hline Hybrid/minimally invasive & 0.66 & 0.32 & 1.38 & .27 \\
\hline
\end{tabular}

Boldface indicates statistical significance.

education early in our initial experience but that improved over time. It is also possible that patients undergoing MIS may have been experiencing delayed complications at home that we did not anticipate early in our experience. Furthermore, we were increasingly using selective rather than routine feeding tubes later in our experience. Because the reasons for most ED visits were feeding tube problems, this may explain lower odds of ED visits later in our experience. In the end, MIS esophagectomy was not associated with repeated or frequent visits and thus it appears that this was a risk factor for transient but not persistent ED use. Another potential reason for lower odds of ED visits in the later study years may be that it reflects the effects of regionalization efforts in our province during 2006 to 2008. During this time, our province began centralizing esophagectomies to a few centers of excellence in each health region. This particular study was focused only on our hospital and thus it was likely hospital-level policies were predominantly the drivers of decreased ED visits over time rather than region-level policies. The exact mechanisms (ie, increased clinic visits and home care) are difficult to delineate from our current study. Further study is required. The act of centralizing esophagectomies may have resulted in a situation where patients are more likely to travel centrally for their esophagectomy but go to their local EDs rather than traveling back to the esophagectomy center. Living outside of the greater metropolitan area of our hospital was independently associated with lower odds of any and frequent ED visits. Sensitivity analysis that included only patients within $100 \mathrm{~km}$ of our hospital replicated our findings. This is not too surprising because these patients are likely to be using ED resources at their closer local hospitals. Wider analyses with the ability to capture ED visits at other, nonindex hospitals are necessary to understand the true burden of ED use after esophagectomy.

Income did not appear to have the expected effect on ED use. We have previously shown that income was not significantly associated with higher readmission rates after esophagectomy (unpublished data). We hypothesize that this reflected the effect of the Canadian universal health care system, where everyone has health insurance. Our finding that higher income was independently associated with higher odds of any ED use but not frequent ED visits is surprising. However, previous studies in Canadian settings have demonstrated that higher-income patients are more likely to access specialist services. ${ }^{11,12}$ In our study, higher-income patients may have been likelier to come to our ED (ie, rather than their local ED or their family physician) to more directly access our specialist care in a prompt manner. Studies in diverse health care settings have also demonstrated such a finding. ${ }^{13}$

Virtually all studies investigating ED use postoperatively focus on 30 days after discharge.$^{10,14-16}$ There is a paucity of data about ED use after esophagectomy with estimates ranging between $13 \%$ and $15 \% .{ }^{17} \mathrm{ED}$ visits within 30 days will likely capture most acute postoperative complications. However, resource use and ED visits continue to occur well beyond the 30-day window and thus a longer time horizon is important to understand the burden of resource use after espohagectomy. The gap in the literature regarding ED use after esophagectomy is important to address. ED visits and readmission are important drivers of health care costs and also likely result in reductions in patient quality of life. There are numerous studies that 
TABLE 3. Baseline characteristics (Frequent emergency department [ED] visits vs no frequent ED visits)

\begin{tabular}{|c|c|c|c|}
\hline Risk factor & $\begin{array}{l}\text { Frequent ED visits } \\
\qquad(n=43)\end{array}$ & $\begin{array}{l}\text { No frequent ED visits } \\
(n=446)\end{array}$ & $P$ value \\
\hline Year of surgery & & & .75 \\
\hline 1999-2006 & $53.5(23)$ & $50.7(226)$ & \\
\hline $2007-2014$ & $46.5(20)$ & $49.3(220)$ & \\
\hline Age at time of surgery $(y)$ & $63.2 \pm 13.3$ & $62.4 \pm 11.6$ & .68 \\
\hline Male sex & $74.4(32)$ & $75.8(338)$ & .85 \\
\hline Median household income (\$) & $69,700(59,586-92,452)$ & $71,889(60,067-88,544)$ & .80 \\
\hline Presence of social support & $79.1(34)$ & $85.2(380)$ & .27 \\
\hline English speaking & $88.4(38)$ & $92.6(413)$ & .36 \\
\hline $\begin{array}{l}\text { Residence distance from index hospital } \\
\text { Within greater metropolitan area } \\
\text { Other city within } 200 \mathrm{~km} \text { of index hospital } \\
\text { Other city } \geq 350 \mathrm{~km} \text { of index hospital }\end{array}$ & $\begin{array}{c}97.7(42) \\
2.3(1) \\
0\end{array}$ & $\begin{array}{c}68.2(304) \\
28.2(126) \\
3.6(16)\end{array}$ & $<.0001$ \\
\hline $\begin{array}{l}\text { Esophagectomy type } \\
\text { Transhiatal } \\
\text { McKeown } \\
\text { Ivor Lewis } \\
\text { Left thoracoabdominal } \\
\text { Pharyngolaryngectomy }\end{array}$ & $\begin{array}{c}18.6(8) \\
9.3(4) \\
44.2(19) \\
25.6(11) \\
2.3(1)\end{array}$ & $\begin{array}{c}15.5(69) \\
21.5(96) \\
36.1(161) \\
25.1(112) \\
1.8(8)\end{array}$ & .30 \\
\hline Lower Charlson comorbidity index* & $74.4(32)$ & $70.2(313)$ & .73 \\
\hline Chemotherapy & $18.6(8)$ & $29.4(131)$ & .16 \\
\hline Radiation therapy & $16.3(7)$ & $26.0(89)$ & .20 \\
\hline Hybrid/minimally invasive & $30.2(13)$ & $21.1(94)$ & .18 \\
\hline Hospital length of stay (d) & $16(12-32)$ & $14(11-21.25)$ & .13 \\
\hline Respiratory complications & $4.6(2)$ & $4.5(20)$ & 1.00 \\
\hline Anastomotic leak & $18.6(8)$ & $15.2(68)$ & .51 \\
\hline Laryngeal nerve complication & $9.3(4)$ & $5.8(26)$ & .32 \\
\hline Thromboembolic complication & $4.6(2)$ & $3.6(16)$ & .67 \\
\hline Cardiac complication & $14.0(6)$ & $11.6(52)$ & .62 \\
\hline Chylothorax & $7.0(3)$ & $5.6(25)$ & .73 \\
\hline
\end{tabular}

Values are presented as $\mathrm{n}(\%)$, mean \pm standard deviation, or median (interquartile range). Boldface indicates statistical significance. ${ }^{*}$ Charlson comorbidity index $0-2$ versus $>3$.

investigate readmissions after esophagectomy, yet there is limited evidence regarding ED visits. ED visits are frequent and can also result in significant health care costs. ED visits that did not result in readmission may have been avoidable. Due to travel time to EDs and time spent waiting to see physicians in EDs, a patient's quality of life is likely to be adversely affected. The influence on quality of life is likely to be amplified the farther away the patient lives from the index hospital. This represents a burden for patients as well as the health care system. Understanding the true burden of and the risk factors for ED visits provides opportunities for designing interventions to try to reduce the monetary system and human costs associated with ED visits.

Because feeding tube issues are the most common cause for presentation to an ED, processes of care addressing feeding tube management appears to provide an opportunity for improved patient outcomes and reduced health care use. Although routine insertion of feeding tubes has been a standard component of esophagectomy, re-evaluation of this practice may be warranted. Several studies have questioned whether routine insertion of feeding tubes after esophagectomy is necessary. ${ }^{18-20}$ Srinathan and colleagues ${ }^{20}$ documented that a substantial number of patients do not require nor benefit from feeding tubes and also reported complications related to feeding tubes. In their study, ${ }^{20}$ they found that $66 \%$ of patients were able start oral full fluid diet by postoperative day 8 and that $6 \%$ of people had feeding tube-related complications (half of which required operative intervention). Thus, they inferred that 66 out of 100 patients were unnecessarily subjected to a risky procedure. ${ }^{20}$ Furthermore, during those first 8 postoperative days, they found that only 
$48 \%$ of the target nutrition was delivered via the feeding tube due to feeding tube cessations in response to abdominal fullness, distension, pain, or diarrhea. ${ }^{20}$ Their findings, in addition to the other literature, prompted us to engage in selective rather than routine use of feeding jejunostomy tubes in our later experience.

A limitation of our study is that it is based on retrospective data. Although data were collected prospectively, this study is subject to the potential biases of retrospective analysis. Another limitation is the inherent value judgment placed on ED visits as a potential marker of poor-quality care. $^{8,21-23}$ It is not clear that ED visits are inappropriate in all cases. In some cases, an ED visit may be quite appropriate and may actually save significant resources by preventing a worsening cascade of complications. Moreover, the push for reducing health resource use by shortening in-hospital LOS after surgery may have the counterproductive effect of increasing resource use if patients are returning to EDs at much higher rates. ${ }^{8,17}$ In our study, we did not find that index hospital LOS was independently associated with increased ED rates. However, our findings about increased odds of ED visits with MIS esophagectomy may have been related to this issue of shorter LOS. A significant limitation is that we only focused on ED visits at our index hospital. Thus, we likely missed ED visits at other hospitals closer to the patients' homes. Wider analyses with the ability to capture ED visits at other, nonindex hospitals are necessary to understand the true burden of ED use after esophagectomy and we are exploring this. Additionally, this study does not include visits to primary care physicians or walk-in clinics. Despite this limitation, our current study allows us to achieve our more important objective, which is to understand the causes of ED use with appropriate granularity so that we may design interventions/solutions to reduce them.

\section{CONCLUSIONS}

There is a high rate of ED use within 1 year of esophagectomy, with $30 \%$ of patients having any ED visits and $8.8 \%$ of patients having frequent visits. Patients living farther away from the index hospital had a lower rate of index hospital ED use; thus, the true rate of ED use is likely higher because these patients are likely utilizing their local hospitals. Some of our findings (higher risk with MIS esophagectomy and lower risk over time) likely reflect the effects of the learning curve of MIS esophagectomy over time. Feeding tube problems are by far the most common reasons for any ED use and frequent ED visits. This is important because these visits are potentially modifiable with preemptive patient/caregiver education or arrangement of clinic visits in lieu of ED use. They could also be potentially comanaged with local health care professionals via telehealth in patients who live far away from the index hospital. These interventions could represent significant cost savings and increased patient quality of life/ satisfaction.

\section{Conflict of Interest Statement}

Authors have nothing to disclose with regard to commercial support.

\section{References}

1. Ajani JA, D'Amico TA, Almhanna K, Bentrem DJ, Besh S, Chao J, et al. Esophageal and esophagogastric junction cancers, Version 1.2015. J Natl Compr Canc Netw. 2015;13:194-227.

2. Kidane B, Coughlin S, Vogt K, Malthaner R. Preoperative chemotherapy for resectable thoracic esophageal cancer. Cochrane Database Syst Rev. 2015;5 CD001556.

3. Atkins BZ, Shah AS, Hutcheson KA, Mangum JH, Pappas TN, Harpole DH Jr, et al. Reducing hospital morbidity and mortality following esophagectomy. Ann Thorac Surg. 2004;78:1170-6.

4. Alanezi K, Urschel JD. Mortality secondary to esophageal anastomotic leak. Ann Thorac Cardiovasc Surg. 2004;10:71-5.

5. Blewett CJ, Miller JD, Young JE, Bennett WF, Urschel JD. Anastomotic leaks after esophagectomy for esophageal cancer: a comparison of thoracic and cervical anastomoses. Ann Thorac Cardiovasc Surg. 2001;7:75-8.

6. Trudel JG, Sulman J, Atenafu EG, Kidane B, Darling GE. Longitudinal evaluation of trial outcome index scores in patients with esophageal cancer. Ann thorac Surg. 2016;102:269-75.

7. Abbott DE, Gaitonde SG, Hanseman DJ, Sutton JM, Wilson GC, Sussman JJ, et al. Resource utilization in esophagectomy: when higher costs are associated with worse outcomes. Ann Surg Oncol 2014; Suppl 1:S34.

8. Chen SY, Molena D, Stem M, Mungo B, Lidor AO. Post-discharge complications after esophagectomy account for high readmission rates. World J Gastroenterol. 2016;22:5246-53.

9. Shah SP, Xu T, Hooker CM, Hulbert A, Battafarano RJ, Brock MV, et al. Why are patients being readmitted after surgery for esophageal cancer? J Thorac Cardiovasc Surg. 2015;149:1384-91.

10. Telem DA, Yang J, Altieri M, Patterson W, Peoples B, Chen H, et al Rates and risk factors for unplanned emergency department utilization and hospital readmission following bariatric surgery. Ann Surg. 2016; 263:956-60

11. Dunlop S, Coyte PC, McIsaac W. Socio-economic status and the utilisation of physicians' services: results from the Canadian National Population Health Survey. Soc Sci Med. 2000;51:123-33.

12. Lemstra M, Mackenbach J, Neudorf C, Nannapaneni U. High health care utilization and costs associated with lower socio-economic status: results from a linked dataset. Can J Public Health. 2009;100:180-3.

13. Makinen M, Waters H, Rauch M, Almagambetova N, Bitran R, Gilson L, et al Inequalities in health care use and expenditures: empirical data from eight developing countries and countries in transition. Bull World Health Organ. 2000;78 $55-65$.

14. Lang BH, Chow FC. Evaluating the incidence, cause, and risk factor or unplanned 30-day readmission and emergency department/general practitioner visit after short-stay thyroidectomy. World J Surg. 2016; 40:329-36.

15. FitzGerald RA, Sehgal AR, Nichols JA, McHenry CR. Factors predictive of emergency department visits and hospitalization following thyroidectomy and parathyroidectomy. Ann Surg Oncol. 2015;22(Suppl 3):S707-13.

16. Hansen DG, Fox JP, Gross CP, Bruun JS. Hospital readmissions and emergency department visits following laparoscopic and open colon resection for cancer. $D i s$ Colon Rectum. 2013;56:1053-61.

17. Lazzarino AI, Nagpal K, Bottle A, Faiz O, Moorthy K, Aylin P. Open versus minimally invasive esophagectomy: trends of utilization and associated outcomes in England. Ann Surg. 2010;252:292-8.

18. Fenton JR, Bergeron EJ, Coello M, Welsh RJ, Chmielewski GW. Feeding jejunostomy tubes placed during esophagectomy: are they necessary? Ann Thorac Surg. 2011;92:504-11.

19. Wheble GA, Benson RA, Khan OA. Is routine postoperative enteral feeding after oesophagectomy worthwhile? Interact Cardiovasc Thorac Surg. 2012;15: 709-12. 
20. Srinathan SK, Hamin T, Walter S, Tan AL, Unruh HW, Guyatt G. Jejunostomy tub feeding in patients undergoing esophagectomy. Can J Surg. 2013; $56: 409-14$

21. Parina RP, Chang DC, Rose JA, Talamini MA. Is a low readmission rate indicative of a good hospital? J Am Coll Surg. 2015;220:169-76.

22. Thomas JW, Holloway JJ. Investigating early readmission as an indicator for quality of care studies. Med Care. 1991;29:377-94.
23. Sundaram A, Srinivasan A, Baker S, Mittal SK. Readmission and risk factors for readmission following esophagectomy for esophageal cancer. J Gastrointest Surg. 2015;19:581-5; discussion 586.

Key Words: esophageal cancer, esophageal surgery, healthcare utilization 